REVESCO. Revista de Estudios Cooperativos

ISSN: $1885-8031$

\title{
Análisis de la actitud emprendedora en sociedades cooperativas pesqueras en México
}

\author{
Linda García Rodríguez ${ }^{1}$, Darío Fuentes Guevara ${ }^{2}$ y Beatriz Pico González $^{3}$ [C
}

Recibido: 11 de marzo de 2020 / Aceptado: 7 de mayo de 2021 / Publicado: 23 de junio de 2021

Resumen. El objetivo del presente artículo fue el conocer cuáles son las normas sociales subjetivas, la actitud y habilidades personales y la intención emprendedora que tienen los presidentes de las cooperativas pesqueras de la Zona Norte del Estado de Sinaloa, México, debido a que se observó que dependiendo de los proyectos que ellos emprendan y de las actividades que ellos realicen, dependerá el usufructo que ellos reciban. La investigación se realizó a través de una metodología de naturaleza cuantitativa con un alcance de acuerdo al nivel de conocimientos obtenidos con dicha investigación: descriptiva - correlacional. Los resultados obtenidos se perfilan a lo escrito en el principio que cita el fomento de la educación cooperativa y de la educación en la economía solidaria, así como a lo que marca la literatura que indica que las persona son capaces de detectar oportunidades de negocio, teniendo la expectativa de éxito y asumiendo los riesgos, así como los rasgos identitarios asentados en factores comunicativos, de cohesión y de relaciones sociales basadas mayormente en la cooperación y solidaridad; por otro lado, se apoya la afirmación de que la viabilidad no sólo depende del emprender colectivo, sino de encontrar las condiciones socio-económicas mínimas que la hagan posible.

Palabras clave: Cooperativas pesqueras; Intención Emprendedora; Actitud; Normas sociales subjetivas; Habilidades personales.

Claves Econlit: B55; M20; M53; O35.

\section{[en] Analysis of the entrepreneurial attitude in cooperative fishing societies in Mexico}

Abstract. The objective of this article was to know what are the subjective social norms, the attitude and personal skills and the entrepreneurial intention of the presidents of the fishing cooperatives of the Northern Zone of the State of Sinaloa, Mexico, because it was observed that depending The usufruct they receive will depend on the projects they undertake and the activities they carry out. The research was carried out through a methodology of a quantitative nature with a scope according to the level of knowledge obtained with said research: descriptive - correlational. The results obtained are outlined to what is written in the principle that cites the promotion of cooperative education and education in the solidarity economy, as well as what the literature indicates that people are capable of detecting business opportunities, having the expectation of success and assuming the risks, as well as the identity traits based on communicative factors, cohesion and social relations based mainly on cooperation and solidarity; on the other hand, the affirmation is supported that the viability not only depends on the collective undertaking, but on finding the minimum socio-economic conditions that make it possible.

Keywords: Fishing cooperatives; Entrepreneurial Intention; Attitude; Subjective social norms; Personal skills.

Sumario. 1. Introducción. 2. Fundamentos Teóricos. 3. Metodología. 4.Resultados y Discusión. 5.Conclusiones. 6. Referencias bibliográficas.

Cómo citar. García Rodríguez, L.; Fuentes Guevara, D.; Pico González, B. (2021) Análisis de la actitud emprendedora en sociedades cooperativas pesqueras en México. REVESCO. Revista de Estudios Cooperativos, vol. 139 , e76632. https://dx.doi.org/10.5209/reve.76632.

\section{Introducción}

La historia que la humanidad ha escrito sobre sí misma desde hace tiempo siempre ha estado marcada por elementos contextuales y sociales que permiten la perduración o el cambio de sistemas sociales acordes que

1 Tecnológico Nacional de México/IT de Los Mochis, México.

Dirección de correo electrónico: linda.gr@mochis.tecnm.mx.

2 Tecnológico Nacional de México/IT de Los Mochis, México.

Dirección de correo electrónico: dario.fg@ mochis.tecnm.mx.

3 Universidad Popular Autónoma del Estado de Puebla, México.

Dirección de correo electrónico: beatriz.pico@upaep.mx. 
permitan la reproducción de la vida colectiva. No obstante, es menester mencionar que dichos contextos cambian con el tiempo.

Hoy, la sociedad actual se encuentra en un proceso de reconfiguración donde la nueva economía es cada vez más global, habiendo cambios vertiginosos en el entorno competitivo, donde los sistemas comerciales se hacen más complejos, los mecanismos y montos de ayuda disminuyen, existiendo así un mayor número de decisiones y de actividades, por lo que demandan de las organizaciones mayor coordinación y control, que exige mayor competitividad, (Salazar, Vargas y Salazar, 2015).

Actualmente el mundo puede verse como una organización compuesta de una gran cantidad de organizaciones, ya sean grandes o pequeñas; por lo que todas las actividades tienen que ver con ellas. Uno de estos grupos de organizaciones son las que se denominan cooperativas, las cuales han contribuido en gran parte al desarrollo económico y social de los países que las han adoptado, (Cabrera, 2015).

De acuerdo con Ban Ki-moon (2015), la desigualdad es un obstáculo para el desarrollo, debido a que priva a las personas de servicios básicos y oportunidades para construir una vida mejor. El modelo cooperativo ayuda a hacer frente a este reto, ya que las cooperativas se esfuerzan por defender los principios de la igualdad y la participación democrática. Se trata de un modelo empresarial construido sobre la base de la inclusión y la sostenibilidad que ofrece un camino hacia la justicia económica, social y política, estando comprometidas con las comunidades a las que prestan servicios.

Se presenta al sistema cooperativo como el tercer camino para enfrentarse a los problemas de la época. "Ya no son el individualismo y el colectivismo los únicos caminos fatales entre los que hay que elegir, la humanidad tiene la esperanza de conservar la libertad humana y la justicia social" (Caballero, Liévano, Longotia y Solis, 2010).

Habría que decir también, que existe un amplio consenso entre varios actores, que apoyan a las cooperativas, entre estos, se tienen organismos de las Naciones Unidas como la Organización Internacional del Trabajo, y la Alianza Cooperativa Internacional, acerca de que la empresa cooperativa es el tipo de organización que mejor satisface todas las dimensiones de la reducción de la pobreza y la exclusión.

Es importante la forma en que las cooperativas ayudan a reducir la pobreza: hallan oportunidades económicas para sus socios; empoderan a las personas desfavorecidas para que defiendan sus intereses; dan seguridad a los pobres permitiéndoles transformar sus riesgos individuales en riesgos colectivos; y median para que sus socios accedan a recursos que les sirvan para ganarse la vida.

Por todo lo anterior, se puede observar que, a nivel nacional e internacional, la cooperativa es considerada un pilar de la economía social, porque aporta "algo más" a la forma de hacer empresa, ya que es un medio de inclusión económica y social.

Es una forma de resolver el problema de la intermediación o la concentración económica que les impide competir en condiciones igualitarias con los grandes concentradores que logran acaparar el mercado, obtener mejores precios en la compra de insumos, lograr economía de escala y poder de negociación en la comercialización de sus productos, (Contreras, Godoy y Alves, 2015); en este contexto, resulta importante utilizar técnicas y estilos de gestión adecuados que les permitan elevar los estándares de eficiencia y elevar los niveles de competencia.

México siendo uno de los principales productores de especies marinas y crustáceos a nivel mundial por su favorable posición geográfica, ya que se encuentra rodeada de los océanos atlántico y pacífico y, debido a la importancia de la pesca en la economía mexicana, el gobierno ha diseñado y puesto en operación programas de financiamiento con orientación a la reactivación de las principales operarias de productos marinos, las sociedades cooperativas, las cuales son reguladas por diversos organismos, tales como: la Procuraduría Federal de Protección al Medio Ambiente (Profepa), Secretaría de Agricultura Ganadería y Desarrollo Rural (SAGARPA) y Comisión Nacional de Acuacultura y Pesca (CONAPESCA). A pesar de que estas puedan financiarse por el apoyo gubernamental, presentan problemas de tipo organizacional y de crecimiento.

Aunque en México sí se pueden encontrar diversos investigadores interesados en el tema de las cooperativas, las cuales son estudiadas desde diferentes aristas (Lara, 2011; Lara y Rico 2011; Alcalá, 2003; Rojas, 2003; Sommers 2008; Contreras y Gómez, 2011 y Torres y Contreras, 2013), ha sido poco abordado el análisis de la intención emprendedora como un mecanismo por medio del cual la sociedad convierte la información técnica en productos y servicios; representando una vía mediante la cual las ineficiencias en el ámbito económico puedan ser solventadas en un tiempo y espacio específico.

Por este motivo, y debido a que el emprendimiento es un término poliédrico y está directamente relacionado con la acción de la persona, la presente investigación busca conocer cuáles son las normas sociales subjetivas, la actitud y habilidades personales y la intención emprendedora que tienen los presidente de las cooperativas de la Zona Norte del Estado de Sinaloa, México, dadas dichas especificidades, verificar con lo que indica la literatura, con la finalidad de acrecentar y desarrollar actividades sustantivas y estratégicas para su crecimiento, y de esta manera perfilarse a lo que se enuncia en la Ley General de Sociedades Cooperativas en el Articulo 6, principio V, que cita el fomento de la educación cooperativa y de la educación en la economía solidaria. 


\section{Fundamentos teóricos}

\subsection{Las cooperativas pesqueras en México}

El término 'cooperativa' designa una "asociación autónoma de personas unidas voluntariamente para satisfacer sus necesidades y aspiraciones económicas, sociales y culturales en común a través de una empresa de propiedad conjunta y de gestión democrática", (OIT, 2014).

El cooperativismo, sin importar qué produzca, es una organización social reconocida a nivel institucional. Según el artículo 2 de la Ley General de Sociedades Cooperativas del 2018 (LGSC), una cooperativa "es una forma de organización social integrada por personas físicas con base en intereses comunes y en los principios de solidaridad, esfuerzo propio y ayuda mutua, con el propósito de satisfacer necesidades individuales y colectivas, a través de la realización de actividades económicas de producción, distribución y consumo de bienes y servicios" (Cámara de Diputados, 2018).

Contreras, Godoy y Alves, (2015) indican que en México operan 15.000 cooperativas aproximadamente, en su mayoría de consumo y producción de bienes y en ellas participan cinco millones de personas, aproximadamente. El sector pesquero en México registra nueve tipos de organizaciones de productores con un total de 5.000 unidades, de las cuales más del 57\% son cooperativas.

\subsubsection{Las cooperativas pesqueras en Sinaloa}

En Sinaloa existen 500 sociedades cooperativas de producción pesquera operadas tanto por pescadores de altamar, como de ribera y de aguas continentales; el total de la fuerza laboral es de 44.802 personas (Trujillo, 2012 y Contreras, et.al., 2015).

Con base en la Ley General de Sociedades Cooperativas (LGSC, 2018), las federaciones de cooperativas podrán agrupar a sociedades cooperativas de la misma rama de la actividad económica. En Sinaloa existen doce federaciones de cooperativas pesqueras que integran a 141 cooperativas pesqueras ribereñas y más de 12.000 pescadores organizados. Sinaloa es un estado que se ubica en el Noroeste de México. Según INEGI (2010) posee 2,767,761 habitantes, de los cuales el 27.2\% habita en comunidades rurales. Es líder en la producción pesquera de México tanto en volumen como en valor; los recursos pesqueros que se capturan en mayor volumen son la sardina, el atún y el camarón, y en valor, el camarón ocupa el primer sitio seguido del atún y la sardina.

En materia de organización pesquera, en Sinaloa se concentra el mayor número de organizaciones cooperativas ribereñas (141) de toda la república, representando el $25 \%$ del total de cooperativas a nivel nacional. Debido a la naturaleza de la actividad y a la estructura sociodemográfica del país (que cuenta con numerosas comunidades rurales), este sector registra una fuerte actividad informal tanto en litorales como en aguas continentales, (Contreras, et.al., 2015).

En relación a la participación de las familias en las actividades económicas de los campos pesqueros, los cooperativistas señalan que la captura del camarón la realizan durante el periodo autorizado por el gobierno (de septiembre a marzo de cada año), y señalan que las esposas se involucran en actividades de apoyo como la recepción de la especie, la toma del peso de la captura y la clasificación del camarón, ya que es necesario separar la especie por su tamaño para su comercialización en los mercados locales, nacionales e internacionales y el empaquetado en plantas congeladoras.

Otro aspecto que se identifica en las cooperativas pesqueras es la tradición del pescador de trasladar de generación en generación el conocimiento del arte de la pesca; los cooperativistas señalan que en la mayoría de las familias la pesca es la principal actividad económica y los padres involucran a los hijos integrándolos como miembros de las cooperativas.

\subsection{El emprendimiento}

El emprendimiento intersecta diversos ejes estratégicos: el crecimiento económico, la equidad, la innovación y el desarrollo productivo. El impulso del emprendimiento es una importante fuente de crecimiento económico y social, pues contribuye a la generación de puestos de trabajo, a la diversificación del tejido productivo, a la innovación, al fortalecimiento del espacio de la pequeña y mediana empresa, al incremento de los niveles de competencia y a una mayor distribución del poder económico, aseguran Acosta, Villacís y Jiménez, (2017).

Según Martinelli (2004) y Acosta, Villacís y Jiménez, (2017), indican que el emprendimiento es un tema frecuente en la literatura económica a partir de mediados del siglo XX, con visión multidisciplinar y su perspectiva deja atrás la idea de la actividad emprendedora espontánea. Esos estudios fundamentalmente basados en la Psicología van más allá de explicar la conducta emprendedora como respuesta al empuje de quedar desempleado o detectar alguna oportunidad de mercado. 
El emprendimiento es la resultante de la articulación de varios factores motivacionales, creencias y del entorno institucional. A nivel global y local se conforman los cambios institucionales, políticos, sociales y económicos que influyen en la disposición a emprender. Los reportes de investigaciones concluyen que la naturaleza y calidad del entorno institucional de un entorno determina la intención a emprender (Urban, 2013; Shane et al., 2003; Kramer, 2005 y Acosta, Villacís y Jiménez, 2017). Por otra parte, se registra que no basta con un entorno institucional favorable y que exista motivación como detonante del proceso de emprendimiento; es necesario que se complemente por actitudes y competencias del individuo que guíen al emprendedor hacia la meta propuesta (De Noble et al., 1999).

La actividad emprendedora es un concepto que se relaciona con los procesos de transformación de las estructuras empresariales y, por ende, tiene impacto en el desarrollo económico y social de las economías nacionales y/o locales. Se mencionan diversos efectos positivos como la diversidad de oferta de productos en el mercado, generación de empleo e incremento de la riqueza (Hernández y Arano, 2015; Encinas, 2018).

También, los emprendedores son agentes que promueven la actividad económica y favorecen ambientes de competencia por medio de la creación de empresas o negocios. Muchas de estas iniciativas emergen en condiciones marginales, y le corresponde al emprendedor hacer uso de sus competencias, redes y actitudes para hacerlas progresar y posicionarlas dentro del mercado de trabajo competitivo determinando de esta manera su éxito o fracaso (Morales et al., 2015). Según Encinas (2018), los emprendedores están comprometidos no sólo con la satisfacción propia, sino además tienen una responsabilidad social para la mejora de su entorno y de la calidad de vida por medio de la generación de empleo.

En la revisión de estudios relacionados con las características claves en el perfil de un futuro emprendedor, es común encontrar la presencia de rasgos psicológicos asociados a la persona que emprende, lo que posibilita, en gran medida, identificar aquellos con altas intenciones de desarrollarse como emprendedor y los que no, opinan que las características psicológicas del emprendedor hacen posible inferir que no existe un perfil único para los emprendedores, sino que la combinación de características de tipo cultural, económico y personal influye más en algunas personas que en otras y, por ende, solo algunos se involucran en el emprendimiento, (Espíritu, 2011; Marulanda, Correa y Mejía, 2009; Duran y Arias, 2016).

\subsubsection{El espíritu emprendedor}

El espíritu de ímpetu empresarial es un poderoso motor de crecimiento y creación de empleo que dota a la comunidad de nuevas capacidades (Lupiáñez y López, 2014). Para poder recuperar el crecimiento y niveles altos de empleo en las zonas pesqueras, es necesario incentivar intensamente el espíritu emprendedor de las sociedades cooperativas.

\subsubsection{Actitud emprendedora}

En las aportaciones de Alda, Villardón y Elexpuro (2012) y de Duran y Arias, (2016), se plantea la necesidad de presentar un perfil como herramienta fundamental para el diseño de la formación de emprendedores, pues esto facilita el establecimiento de metas de formación. Entre las características de dicho perfil, los autores recomiendan desarrollar: autonomía, autoconfianza, asertividad, creatividad, optimismo, confianza, entre otros. Estas características son distintivas de la actitud emprendedora y, aunque tradicionalmente los estudios relacionados con la identificación del perfil emprendedor utilizan de forma indistinta los constructos autoeficacia y actitud emprendedora; la investigación de Durán-Aponte (2013) resulta un intento exitoso para diferenciar entre ambos constructos, a través de un estudio de validez de constructo. La autora afirma que la autoeficacia emprendedora se refiere al juicio que hacen las personas sobre su capacidad de emprender un negocio; en cambio la actitud emprendedora considera que la persona deberá ser optimista, proactiva, persistente y creativa, lo cual está más relacionado con su personalidad.

\subsubsection{Las normas subjetivas}

La norma subjetiva es un razonamiento que pone de manifiesto la presión social percibida por la persona que va a realizar la conducta. Muestra el resultado de la evaluación que la persona hace si los otros significativos (familiares o amigos) piensan que él o ella realicen la conducta y dé las motivaciones para ajustarse a esas expectativas (Ajzen, 1991; Guzmán, Llamas, Rodríguez y Alonso, 2014).

Es por esto por lo que, resulta relevante poder determinar si en los miembros de las cooperativas pesqueras existen determinados indicadores que contribuyan a ver si cuentan con actitudes emprendedoras, con la finalidad de acrecentar y desarrollar actividades sustantivas y estratégicas para su crecimiento. 


\subsection{Negocio inclusivo}

El concepto de Negocios Inclusivos ha sido formulado principalmente por organizaciones internacionales y colombianas (Fundación AVINA, 2008; Gradl y Knobloch, 2010; PNUD - Programa de las Naciones Unidas para el Desarrollo, 2010; SNV - Servicio Holandés de Cooperación al Desarrollo y CECODES - Consejo Empresarial Colombiano para el Desarrollo, 2008; SNV - Servicio Holandés de Cooperación al Desarrollo y WBCSD - Consejo Mundial Empresarial para el Desarrollo Sostenible, 2008; WBCSD - Concejo Mundial Empresarial para el Desarrollo Sostenible, 2006), mostrando que los negocios inclusivos tienen origen en la práctica empresarial de los sectores productivos, Maestre, Páez, Mesías y Lombana (2019).

A continuación, en la Tabla 1, se muestran algunas de las definiciones más relevantes que han sido utilizadas para explicar el concepto de negocios inclusivos:

Tabla. 1. Concepto de Negocios Inclusivos

\begin{tabular}{|c|c|}
\hline Concepto & Autor institucional \\
\hline $\begin{array}{l}\text { Iniciativas económicamente rentables, ambiental y } \\
\text { socialmente responsables, que utilizan los mecanismos del } \\
\text { mercado para mejorar la calidad de vida de personas de } \\
\text { bajos ingresos }\end{array}$ & Fundación AVINA, (2008) \\
\hline $\begin{array}{l}\text { Iniciativa empresarial que sin perder de vista el objetivo de } \\
\text { generar ganancias, contribuye a la superación de la pobreza } \\
\text { a través de la incorporación de ciudadanos de menores } \\
\text { recursos a la cadena de valor }\end{array}$ & $\begin{array}{c}\text { SNV - Servicio Holandés de Cooperación al } \\
\text { Desarrollo y WBCSD - Consejo Mundial } \\
\text { Empresarial para el Desarrollo Sostenible } \\
(2008: 2)\end{array}$ \\
\hline $\begin{array}{l}\text { Agregar valor en los negocios por igual tanto para las } \\
\text { empresas como para las personas que viven en la pobreza. }\end{array}$ & Gradl y Knobloch (2010:4) \\
\hline
\end{tabular}

Fuente: Maestre, Páez, Mesías y Lombana (2019).

De acuerdo con Maestre, et. al., (2019), el origen del término negocios inclusivos está relacionado con los trabajos realizados por Prahalad y Hart (2002) que estudiaron la situación de las personas con bajo poder adquisitivo (denominados base de la pirámide). Los cuales presentan la base de la pirámide como un mercado altamente interesante para las grandes empresas, ya que a través de la transformación de sus productos logran crear bienes con buenas características y aumentando la participación en el mercado.

\section{Metodología}

El presente estudio se abordó con una metodología enfocada en Hernández y Mendoza, (2018), siendo una investigación de naturaleza cuantitativa no dejando atrás el aspecto cualitativo debido a que proporcionó profundidad a los datos, con un alcance de acuerdo con el nivel de conocimientos obtenidos con dicha investigación: descriptiva - correlacional.

\subsection{Elección y tipo de muestra}

Hay que mencionar que, de acuerdo al registro de la Confederación Nacional Mexicana de Cooperativas Pesqueras y Acuícolas en la Zona Norte de Sinaloa, solo está afiliada la Sociedad Cooperativa "Federación de Cooperativas Pesqueras del Siglo 21, S.C. de R.L."; sin embargo, visiblemente existen otras que no forman parte de esta Confederación pero que si están constituidas como federaciones y también dan vida a la actividad pesquera.

Es por ello, que se determinó realizar el estudio en las organizaciones formales con denominación de Federación que agrupa a Sociedades Cooperativas de Producción Pesqueras (S.C.P.P.), establecidas en la ciudad de Los Mochis, Sinaloa, con concesión o permiso de pesca de camarón, siendo esta ciudad un punto central en la economía pesquera del Norte del país por su ubicación y acceso a diversos medios de transporte que favorecen el desarrollo de la actividad pesquera.

Unidad de Análisis: Presidentes de las Cooperativas pesqueras

Para la selección de la población de la investigación, se consideró a la Federación Regional de Sociedades Cooperativas de la industria pesquera "Norte de Sinaloa Sur de Sonora" S.C. de R.L., ya que está cuenta con 
mayor antigüedad en la ciudad de Los Mochis, Sinaloa, (desde 1968 hasta la fecha), y la cual agrupa a 51 Sociedades Cooperativas.

Resulta relevante mencionar que todas estas Sociedades Cooperativas, han obtenido algunas de las siguientes concesiones: captura de camarón, escama, jaiba, almeja, almeja pata de mula, callo de hacha, calamar gigante, tiburón, agua mala y tilapia.

Para el estudio realizado, de todas estas cooperativas se descartaron las que fueron dadas de baja (9), las que están en Sonora (12) o colindan con Sonora (2), las que tienen un mismo presidente (1) y las granjas camaroneras pero que no son propiamente cooperativas (5). Quedando finalmente un total de 22 Sociedades Cooperativas siendo estás a las que se les aplicó el instrumento de medición y las cuales se encuentran establecidas en los siguientes campos pesqueros: Topolobampo, Bachomobampo 2, El Colorado, El Aguajito, Las Lajitas, El jitzamuri y Bacorehuis; destacando que entre ellas, la que está conformada por un menor número de socios es Las Lajitas (5) y la que tiene un mayor número de socios se encuentra en Paredones (496); ambas trabajan en la zona norte del Estado de Sinaloa.

De las 22 Sociedades Cooperativas anteriormente mencionadas y seleccionadas, 14 tienen las concesiones de camarón y solo 8 tienen la concesión de jaiba.

\subsection{Selección del Instrumento}

Se aplicó un instrumento de medición de tipo cuantitativo, mediante el uso de un cuestionario estructurado, por ende, toda la información que se recabó fue de forma explícita y estandarizada. El cuestionario utilizado se basó en Pico, Vidal, Tapia y Mendoza (2019), para el análisis de actitudes e indicadores sobre emprendimiento que muestran los presidentes de las cooperativas pesqueras, el cual fue estructurado de la siguiente manera:

a) Normas sociales subjetivas

b) Actitud y habilidades personales

c) Intención emprendedora

La escala empleada en el cuestionario fue de tipo Likert (Hernández y Mendoza, 2018), conformado por 7 puntos, los cuales van de totalmente de acuerdo a totalmente en desacuerdo, determinando su grado de confiabilidad mediante el Alfa de Cronbach.

\subsection{Presentación, análisis e interpretación de resultados}

Para la indagación de los datos del presente estudio, se planteó un análisis descriptivo y correlacional del conjunto de variables extraídas que permiten conocer algunas características de la muestra relacionadas con las normas sociales subjetivas, la actitud y habilidades personales y la intención emprendedora, con la finalidad de obtener un perfil global de los presidentes de las cooperativas pesqueras en estudio.

\section{Resultados y Discusión}

\subsection{Resultados descriptivos}

De los resultados obtenidos del cuestionario, aplicado a los presidentes de cooperativas pesqueras del Norte de Sinaloa, se pudo observar en primera instancia, que el $90.91 \%$ de los presidentes encuestados tienen interés de iniciar un nuevo proyecto; el $81.82 \%$ consideran tener ideas novedosas; con un $63.64 \%$ indican que durante años tuvieron una empresa, la cual funcionó adecuadamente, además de contar con ideas novedosas; y a su vez, el $45.45 \%$ de los presidentes exponen el haber emprendido y no haberles funcionado. De manera que, como primer resultado se puede inferir en que los presidentes cuentan con indicadores que les permite tener una actitud emprendedora.

A continuación, se presentan otros resultados de la investigación; la información es de carácter estadístico descriptivo del conjunto de variables extraídas del cuestionario, el cual permite conocer algunas características de la muestra. Para efectos de una mejor interpretación de resultados, se dividió en tres el análisis del cuestionario, todo esto de acuerdo con el tipo de preguntas que se realizaron. 
Tabla. 2. Tabla de respuestas con respecto a las normas sociales subjetivas

\begin{tabular}{|l|c|c|c|c|}
\hline \multicolumn{1}{|c|}{ Normas Sociales Subjetivas } & $\begin{array}{c}\text { Totalmente } \\
\text { de acuerdo } \\
\%\end{array}$ & $\begin{array}{c}\text { Muy de } \\
\text { acuerdo } \\
\%\end{array}$ & $\begin{array}{c}\text { De } \\
\text { acuerdo } \\
\%\end{array}$ & $\begin{array}{c}\text { Totalmente de } \\
\text { acuerdo/Muy de } \\
\text { acuerdo/ De } \\
\text { acuerdo \% }\end{array}$ \\
\hline $\begin{array}{l}\text { Mi familia me apoya en mi decisión por } \\
\text { emprender }\end{array}$ & 95.45 & 4.55 & 0.00 & $\mathbf{1 0 0 . 0 0}$ \\
\hline $\begin{array}{l}\text { Mis amigos me animan para realizar } \\
\text { emprendimientos }\end{array}$ & 86.36 & 4.55 & 4.55 & $\mathbf{9 5 . 4 6}$ \\
\hline $\begin{array}{l}\text { Considero que las instituciones de gobierno } \\
\text { apoyan nuevos emprendimientos }\end{array}$ & 72.73 & 4.55 & 9.09 & $\mathbf{7 7 . 2 7}$ \\
\hline $\begin{array}{l}\text { Cuento con información de los expertos para } \\
\text { madurar mis ideas hacia el emprendimiento }\end{array}$ & 40.91 & 27.27 & 9.09 & $\mathbf{7 7 . 2 7}$ \\
\hline $\begin{array}{l}\text { Cuento con información suficiente para iniciar } \\
\text { nuevos emprendimientos }\end{array}$ & 40.91 & 31.82 & 9.09 & $\mathbf{7 2 . 7 3}$ \\
\hline $\begin{array}{l}\text { Creo que las instituciones educativas apoyan la } \\
\text { acción de emprender }\end{array}$ & 68.18 & 9.09 & 13.64 & $\mathbf{9 0 . 9 1}$ \\
\hline $\begin{array}{l}\text { Pienso que los emprendimientos contribuyen al } \\
\text { crecimiento económico de una región }\end{array}$ & 81.82 & 4.55 & 9.09 & $\mathbf{8 6 . 3 6}$ \\
\hline $\begin{array}{l}\text { Existen ayuda de diferentes instituciones al inicio, } \\
\text { durante y al final de nuevos emprendimientos }\end{array}$ & 54.55 & 4.55 & 13.64 & $\mathbf{7 2 . 7 3}$ \\
\hline $\begin{array}{l}\text { Creo que la sociedad valora a los emprendedores } \\
\text { con niveles altos }\end{array}$ & 68.18 & 13.64 & 4.55 & $\mathbf{8 1 . 8 2}$ \\
\hline $\begin{array}{l}\text { Dispongo de una red de contactos, entre ellos } \\
\text { otros emprendedores, que me facilitan } \\
\text { conocimientos y ayuda para emprender }\end{array}$ & 59.09 & 13.64 & 18.18 & $\mathbf{9 0 . 9 1}$ \\
\hline
\end{tabular}

Fuente: Elaboración propia

La tabla anterior representa las frecuencias y porcentajes de las respuestas con opinión de "Totalmente de acuerdo, Muy de acuerdo y De acuerdo" del total de respuestas del primer bloque de preguntas, las cuales ayudaron a conocer la opinión que los presidentes de las cooperativas pesqueras tienen sobre normas sociales subjetivas, considerando que sus familias los apoyan y los amigos los animan a emprender, representado con un porcentaje del $100 \%$ y del $95.46 \%$ respectivamente; con un $90.91 \%$ indican que las instituciones educativas apoyan el emprendimiento y que disponen de una red de contactos que les facilita conocimientos y ayuda para poder emprender.

La Tabla 3 representa las frecuencias y porcentajes de las respuestas con opinión de "Totalmente de acuerdo y Muy de acuerdo" del total de respuestas del segundo bloque de preguntas, las cuales ayudaron a saber la opinión que los presidentes de las cooperativas pesqueras tienen sobre su actitud y habilidades personales; observando con un $100 \%$ que los presidentes consideran que el confiar en sus decisiones les lleva a un buen fin al emprender algo además de estar comprometidos y capacitado/as para iniciar una nueva actividad y sacarla adelante; con un porcentaje del $95.45 \%$ coinciden en decir que son optimistas cuando quieren emprender ante los momentos críticos y difíciles, consiguiendo los resultados propuestos; considerando así que el emprender algo nuevo les proporcionaría una mayor seguridad y estabilidad económica, tanto a ellos, como a sus familias; además de considerar que su lema es "no claudicar": levantarme y seguir adelante, el cual queda representado con un $90.91 \%$ del total de respuestas. 
Tabla. 3. Tabla de respuestas con respecto a la actitud y habilidades personales

\begin{tabular}{|c|c|c|c|c|}
\hline ACTITUD Y HABILIDADES PERSONALES & $\begin{array}{c}\text { Totalmente } \\
\text { de acuerdo } \\
\%\end{array}$ & $\begin{array}{l}\text { Muy de } \\
\text { acuerdo } \\
\%\end{array}$ & $\begin{array}{c}\text { De acuerdo } \\
\%\end{array}$ & $\begin{array}{c}\text { Totalmente } \\
\text { de acuerdo } \\
\text { /Muy de } \\
\text { acuerdo } \\
\%\end{array}$ \\
\hline $\begin{array}{l}\text { Confío en que mis decisiones me lleven a un buen } \\
\text { fin al emprender algo }\end{array}$ & 95.45 & 4.55 & 0.00 & 100.00 \\
\hline $\begin{array}{l}\text { Soy optimista cuando quiero emprender ante los } \\
\text { momentos críticos y difíciles }\end{array}$ & 86.36 & 9.09 & 0.00 & 95.45 \\
\hline $\begin{array}{l}\text { Construyo relaciones sociales como una prioridad } \\
\text { para desarrollar acciones de emprendimiento }\end{array}$ & 72.73 & 0.00 & 0.00 & 72.73 \\
\hline $\begin{array}{l}\text { Me conduzco con actitud de servicio en el } \\
\text { emprendimiento de mis proyectos }\end{array}$ & 81.82 & 0.00 & 0.00 & 81.82 \\
\hline $\begin{array}{l}\text { Tengo la plena seguridad que nunca es tarde para } \\
\text { empezar de nuevo en algo diferente }\end{array}$ & 86.36 & 0.00 & 0.00 & 86.36 \\
\hline $\begin{array}{l}\text { Mi lema es "no claudicar": levantarme y seguir } \\
\text { adelante }\end{array}$ & 86.36 & 4.55 & 0.00 & 90.91 \\
\hline $\begin{array}{l}\text { Soy una persona comprometida: si tuviese } \\
\text { oportunidad y los recursos me encantaría } \\
\text { emprender algo nuevo }\end{array}$ & 95.45 & 4.55 & 0.00 & 100.00 \\
\hline $\begin{array}{l}\text { Reconozco mis debilidades y trabajo en ellas para } \\
\text { convertirlas en fortalezas }\end{array}$ & 77.27 & 9.09 & 0.00 & 86.36 \\
\hline $\begin{array}{l}\text { Me considero capacitado/a para iniciar una nueva } \\
\text { actividad y sacarla adelante }\end{array}$ & 81.82 & 18.18 & 0.00 & 100.00 \\
\hline $\begin{array}{l}\text { Ser emprendedor (crear algo nuevo) me aportaría } \\
\text { una gran satisfacción personal }\end{array}$ & 81.82 & 4.55 & 0.00 & 86.36 \\
\hline $\begin{array}{l}\text { Creo que si emprendiera algo nuevo conseguiría } \\
\text { los resultados que me propusiese }\end{array}$ & 90.91 & 4.55 & 0.00 & 95.45 \\
\hline $\begin{array}{l}\text { En el caso que emprendiera, no tengo miedo a } \\
\text { fracasar o a quedar en ridículo }\end{array}$ & 77.27 & 4.55 & 0.00 & 81.82 \\
\hline $\begin{array}{l}\text { Emprender algo nuevo me proporcionaría una } \\
\text { mayor seguridad y estabilidad económica, tanto a } \\
\text { mi como a mi familia }\end{array}$ & 95.45 & 0.00 & 0.00 & 95.45 \\
\hline
\end{tabular}

Fuente: Elaboración propia. 
Tabla. 4. Tabla de respuestas con respecto a la intención emprendedora

\begin{tabular}{|l|c|c|c|c|}
\hline \multicolumn{1}{|c|}{ INTENCIÓN EMPRENDEDORA } & $\begin{array}{c}\text { Totalmente } \\
\text { de acuerdo } \\
\%\end{array}$ & $\begin{array}{c}\text { Muy de } \\
\text { acuerdo } \\
\%\end{array}$ & $\begin{array}{c}\text { De acuerdo } \\
\%\end{array}$ & $\begin{array}{c}\text { Totalmente } \\
\text { de } \\
\text { acuerdo/Muy } \\
\text { de acuerdo \% }\end{array}$ \\
\hline $\begin{array}{l}\text { Estoy interesado/a en emprender alguna } \\
\text { actividad diferente a lo que hago actualmente }\end{array}$ & 77.27 & 0.00 & 0.00 & $\mathbf{7 7 . 2 7}$ \\
\hline $\begin{array}{l}\text { Tengo plena seguridad que emprender en algo } \\
\text { es lo mejor para superarme }\end{array}$ & 77.27 & 9.09 & 0.00 & $\mathbf{8 6 . 3 6}$ \\
\hline $\begin{array}{l}\text { Cuento con ideas creativas para iniciar una } \\
\text { nueva actividad }\end{array}$ & 63.64 & 22.73 & 0.00 & $\mathbf{8 6 . 3 6}$ \\
\hline $\begin{array}{l}\text { Mis decisiones me conducen a ser un } \\
\text { emprendedor por naturaleza }\end{array}$ & 63.64 & 18.18 & 0.00 & $\mathbf{8 1 . 8 2}$ \\
\hline $\begin{array}{l}\text { Estoy seguro/a que el romper los paradigmas } \\
\text { actuales me lleva a una situación mejor }\end{array}$ & 50.00 & 27.27 & 0.00 & $\mathbf{7 7 . 2 7}$ \\
\hline $\begin{array}{l}\text { Necesito transformar mis ideas en actuaciones } \\
\text { concretas que me conducen a emprender }\end{array}$ & 68.18 & 27.27 & 0.00 & $\mathbf{9 5 . 4 5}$ \\
\hline $\begin{array}{l}\text { Me gusta aportar nuevas ideas como } \\
\text { empleado para mejorar la organización donde } \\
\text { trabajo }\end{array}$ & 86.36 & 9.09 & 0.00 & $\mathbf{9 5 . 4 5}$ \\
\hline $\begin{array}{l}\text { Me interesa emprender bajo esquemas no } \\
\text { convencionales }\end{array}$ & 50.00 & 13.64 & 0.00 & $\mathbf{6 3 . 6 4}$ \\
\hline $\begin{array}{l}\text { He pensado seriamente en emprender, en } \\
\text { iniciar algo nuevo y diferente }\end{array}$ & 72.73 & 18.18 & 0.00 & $\mathbf{9 0 . 9 1}$ \\
\hline
\end{tabular}

Fuente: Elaboración propia

La tabla anterior representa las frecuencias y porcentajes de las respuestas con opinión de "Totalmente de acuerdo y Muy de acuerdo" del total de respuestas del tercer bloque de preguntas, las cuales ayudaron a conocer la opinión que los presidentes de las cooperativas pesqueras tienen sobre su intención emprendedora, considerando así que necesitan transformar sus ideas en actuaciones concretas que los conduzcan a emprender, ya que les gusta aportar nuevas ideas como empleado para mejorar la organización donde trabajan, lo cual queda representado con un $95.45 \%$; por otra parte, con un $90.91 \%$ indican haber pensado seriamente en emprender, en iniciar algo nuevo y diferente; así mismo, consideran que tienen plena seguridad que emprender en algo es lo mejor para superarse, ya que indican contar con ideas creativas para iniciar una nueva actividad, lo cual se representa con un $86.36 \%$ del total de respuestas.

\subsection{Resultados del Análisis de Correlación de Pearson}

La razón de considerar el coeficiente de correlación fue para identificar la fuerza de la relación entre variables normas sociales subjetivas, actitud y habilidades e intensión emprendedoras, en el sentido positivo; por lo que para el presente estudio fue a partir de $r \geq 0.7$; el valor significa que las variables presentan una relación positiva considerable.

Interpretación de las variables que mostraron mayor coeficiente de correlación.

Tabla. 5. Interpretación del Coeficiente de Correlación de las variables: Normas sociales subjetivas y Actitud y habilidad emprendedora

\section{Correlación entre Variables}

$(+/-)$ :

Normas sociales subjetivas y Actitud y habilidad emprendedora

\begin{tabular}{l||c}
\hline Resultado 4 pares de datos & \multicolumn{1}{c}{\begin{tabular}{c} 
4 correlación positiva considerable \\
\hline Nivel de Confianza
\end{tabular}} \\
\hline Conclusión & $\begin{array}{l}\text { En este sentido, se puede notar que entre estas dos variables existen } 4 \\
\text { correlaciones con un 99\% de confianza, entre las cuáles se puede } \\
\text { mencionar: }\end{array}$ \\
\hline \hline
\end{tabular}




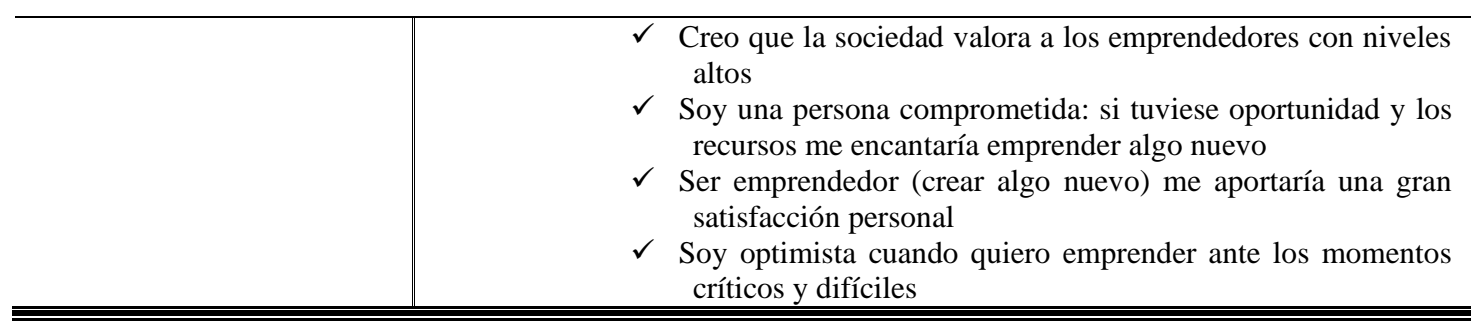

Fuente: Elaboración propia

Tabla. 6. Interpretación del Coeficiente de Correlación de las variables: Normas sociales subjetivas e intensión emprendedora

\begin{tabular}{|c|c|}
\hline \multicolumn{2}{|c|}{$\begin{array}{l}\text { Correlación entre Variables } \\
\qquad(+/-) \text { : }\end{array}$} \\
\hline \multicolumn{2}{|c|}{ Normas sociales subjetivas e intensión emprendedora } \\
\hline Resultado 4 pares de datos & 4 correlación positiva considerable \\
\hline Nivel de Confianza & 4- $99 \%$ \\
\hline Conclusión & $\begin{array}{l}\text { - En este sentido, se puede notar que entre estas dos variables } \\
\text { existen } 3 \text { correlaciones con un } 99 \% \text { de confianza, entre las cuáles } \\
\text { se puede mencionar: } \\
\checkmark \quad \text { Mis decisiones me conducen a ser un emprendedor por } \\
\text { naturaleza } \\
\checkmark \quad \text { Soy optimista cuando quiero emprender ante los } \\
\text { momentos críticos y difíciles } \\
\checkmark \quad \text { Mi familia me apoya en mi decisión por emprender } \\
\checkmark \quad \text { Tengo plena seguridad que emprender en algo es lo } \\
\text { mejor para superarme }\end{array}$ \\
\hline
\end{tabular}

Fuente: Elaboración propia

Está investigación permitió aplicar empíricamente los conceptos de norma sociales subjetivas, actitud y habilidades e intención emprendedoras en los presidentes de las cooperativas pesqueras del Norte de Sinaloa.

Hay que destacar que los presidentes si les dan importancia a sus familiares, amigos y a las figuras de autoridad a la hora de tomar un comportamiento. Así mismo, se observa que son personas dispuestas a asumir riesgos con gran tolerancia, lo que implica tener menos miedo en auto-emplearse y/o crear su propio emprendimiento. Otro rasgo importante es la deseabilidad, la viabilidad percibida y la propensión a actuar.

De acuerdo con lo anterior, se puede indicar que los presidentes de las cooperativas pesqueras han demostrado ser efectivos como predictores de la intención emprendedora, buscando la oportunidad y comprometiéndose rápidamente; están dispuestos a involucrarse y ejecutar procesos de cambio con responsabilidad ante diferentes escenarios en virtud de las modificaciones continuas en el entorno.

Dicho lo anterior, se puede indicar que se comprueba lo que indica García, Villaseca y González, (2019), al decir que cualquier emprendedor presentan una serie de características que los definen, siendo capaces de detectar oportunidades de negocio, teniendo la expectativa de éxito y asumiendo los riesgos de un contexto incierto (económicos, financieros, tecnológicos...).

Por otro lado, en publicaciones que analizan a las empresas recuperadas por sus trabajadores como parte de la economía social y solidaria, autores como (Altuna, 2019; Santos Araújo et al, 2019; Razeto, 2007; Guerra, 2007; Singer, 2007; Coraggio, 2018, 2011; Hinkelammert, 2009; Cruz, 2011), coinciden en caracterizar los emprendimientos de dicho sector según dos aspectos centrales que los diferencian de las empresas tradicionales: por una parte, plantean que en el plano de la producción, el elemento sustancial estaría dado por el factor de trabajo y las capacidades de trabajo por sobre el capital; y por otra parte, resaltan el rasgo identitario asentado en factores comunicativos, la cohesión y relaciones sociales basadas mayormente en la cooperación y solidaridad; esto último confirma la importancia de haber analizado las normas sociales subjetivas, la actitud y habilidades personales y la intención emprendedora, de los presidentes de las cooperativas en estudio, y con esto hacerles ver que existen formas de poder sobresalir en un entorno competitivo, y esto en función de que la distribución de los rendimientos es en proporción a la participación de los socios, y observando que dependiendo de los proyectos que ellos emprendan y de las actividades que ellos realicen, dependerá el usufructo que ellos reciban, por lo que resulta aún más relevante, el considerar este impulso dentro de este tipo de organizaciones.

Considerando al mismo tiempo y no menos importante, lo que indica Rieiro (2020): 
"La recuperación de un emprendimiento productivo puede entenderse como una nueva forma de acción colectiva. De esta manera, durante la crisis socioeconómica vivida a principios de siglo, algunos/as trabajadores/as han desafiado al desempleo y demostrado que algunas empresas de "capitales en crisis" pueden sobrevivir al ser gestionadas por los/as propios/as trabajadores/as. Sin embargo, muchas iniciativas no logran consolidarse, dado que la viabilidad no sólo depende del "emprender" colectivo, sino de encontrar las condiciones socio-económicas mínimas que la hagan posible”.

\section{Conclusiones}

El objetivo del presente artículo fue el conocer cuáles son las normas sociales subjetivas, la actitud y habilidades personales y la intención emprendedora que tienen los presidentes de las cooperativas pesqueras de la Zona Norte del Estado de Sinaloa, México, obteniendo como resultados aspectos interesantes, tales como, la mayoría posee interés en iniciar un nuevo proyecto, cuentan con ideas novedosas y han contado con empresas en algún momento de su vida.

La contribución relevante obtenida del análisis del conocimiento de las normas sociales subjetivas, la actitud y habilidades personales y la intención emprendedora se centra en el conocimiento de aspectos de gran importancia que radican en las personas con iniciativa emprendedora, como lo son: la familia, los amigos e instituciones educativas que apoyan el emprendimiento y facilitan el conocimiento del mismo; la confianza en sus propias decisiones, el optimismo ante los momentos críticos y difíciles obteniendo buenos resultados, considerar al emprendimiento como algo que brinda mayor seguridad y estabilidad económica; así como la aportación de nuevas ideas, el pensar en iniciar algo nuevo y diferente, así como el considerar que emprender es lo mejor para superarse. Todo esto derivado del uso de una metodología cuantitativa a través de un análisis descriptivo y correlacional entre las variables de estudio.

Como consecuencia de lo anterior, se puede inferir en que los presidentes de las sociedades cooperativas en estudio cuentan con indicadores que les permite tener una actitud emprendedora, lo cual permite concluir que resulta factible y de suma importancia impulsar la actitud y el espíritu emprendedor en los integrantes de las sociedades cooperativas pesqueras del Norte de Sinaloa, siendo estas de gran impulso para la economía y una de las más importantes a nivel nacional.

Por lo anterior, se concluye que las cooperativas pesqueras del Norte de Sinaloa se perfilan a lo que indica la Ley General de Sociedades Cooperativas con respecto a lo que indica el Artículo 6.- Las sociedades cooperativas deberán observar en su funcionamiento el principio (V) que cita el fomento de la educación cooperativa y de la educación en la economía solidaria para que aumenten su productividad y eficiencia.

Por otro lado, se considera relevante continuar con un estudio que permita el análisis de la actitud y aptitud innovadora y creativa que tienen los presidentes de las cooperativas pesqueras, a través de aspectos importantes como el pensamiento creativo y la innovación disruptiva, con la finalidad de contar con un panorama completo con respecto a la identificación de sus capacidades para resolver problemas creativamente y las de convertir nuevas posibilidades en realidades, usando un particular pensamiento crítico y aplicando una adecuada actitud y aptitud.

Todo ello, con el objeto de reconocer que el buen uso de la interconexión sistémica que existe entre todos estos eslabones involucrados es primordial para la identificación y aprovechamientos de necesidades y que si se llevan a cabo a través de objetivos claramente fijados y junto a una metodología diseñada específicamente para cumplirlos serian favorables en beneficio de las sociedades cooperativas pesqueras del Norte de Sinaloa.

Así mismo, se sugiere abordar la investigación a través de la Teoría del Comportamiento Planificado, la cual describe a las intenciones emprendedoras como el mejor predictor del emprendimiento futuro, lo que permitiría tener otra arista del estudio.

\section{Referencias bibliográficas}

Acosta, V. M.A., Villacís. A, W.H. y Jiménez, C. M.E. (2017). Factores que conforman la intención emprendedora de estudiantes de la Universidad de Guayaquil. Revista Científica. Dominio de las Ciencias. Vol. 3, No 3, pp. 11161146. ISSN 2477-8818. https://dialnet.unirioja.es/servlet/articulo?codigo $=6244045$.

Alcalá, G. (2003) Políticas Pesqueras en México. 1946 - 2000. Contradicciones y Aciertos en la Planificación de la Pesca Nacional. México: COLMEX, CICESE y POLMICH. https://biblioteca.cicese.mx/libros/politicas/pesqueras.pdf.

Altuna Gabilondo, L. (2019) El valor de la solidaridad en clave de creatividad. Otra Economía, Vol. 12, № 21, pp. 153 167.

Ajzen I. (1991) The Theory of planned behavior. Organizational Behavior and Human Decision Process 1991; 50, pp. 179-211. 
Alda, V., R., Villardón, Gallego. , L. y Elexpuro, A., I. (2012) Propuesta y validación de un perfil de competencias de la persona emprendedora. Implicaciones para la formación. Electronic Journal of Research in Educational, (28), pp. 1057-1080. Recuperado de: https://www.redalyc.org/pdf/2931/293124654006.pdf.

Ban Ki-moon, (2015) ONU destaca la importancia de las cooperativas para el futuro sostenible. Noticias ONU. Parr.2. Recuperado de https://news.un.org/es/story/2015/07/1334101.

Caballero, H. L., Liévano, M.D.J.M., Longoria, J.R.T., y Solis, J.I.C., (2010) Titulo de la ponencia: "Riesgos actuales de una sociedad cooperativa de producción pesquera en Chiapas". Recuperado de: https://1library.co/document/zk7kxp1q-ponencia-riesgos-actuales-sociedad-cooperativa-produccion-pesquerachiapas.html.

Cabrera. R., A.K. (2015) México: Análisis documental del impacto socioeconómico en comunidades rurales. Strategy, Technology \& Society. Vol 1. No. 1.

Cámara de Diputados (2018) Ley Federal de Sociedades Cooperativas (última Reforma DOF 13-08-2018). Formato Digital (PDF) descargado del sitio web http://www.diputados.gob.mx/LeyesBiblio/pdf/143 190118.pdf.

Contreras, M. R. y Gómez, P. (2011) La asociación y la toma de decisiones en la organización cooperativa. Ponencia titulada en el congreso internacional Rulesccop: Soluciones de la Economía Social y solidaria a un mundo en crisis. San Gil, Santander. Colombia. Recuperado en: https://www.economiasolidaria.org/buscadoravanzado/?.sf_s=ponencia\%20La\%20asociaci\%C3\%B3n\%20y\%201a\%20toma\%20de\%20decisiones\%20en\%201a \%20organizaci\%C3\%B3n\%20cooperativa.\%20.

Contreras, L., M.R., Godoy, A.E., y Alves, L.F. (2015) Las cooperativas de Brasil-México-Argentina y el desarrollo. In: Pasado, presente y futuro de las regiones en México y su estudio. Asociación Mexicana de Ciencias para el Desarrollo Regional A.C., México. ISBN AMECIDER: 978-607-96649-1-6 UNAM-IIEc: 978-607-02-743.

Coraggio, J. L. (2011) Principios, instituciones y prácticas de la economía social y solidaria. En: Alberto A. y Martínez E. (Ed) Economía social y solidaria. El trabajo antes que el capital. Quito: AbyaYala, pp. 1-25.

Coraggio, J.L. (2018) Potenciar la Economía Popular Solidaria: una respuesta al neoliberalismo. Otra Economía. Vol $11, \mathrm{~N}^{\mathrm{o}} 20, \mathrm{pp} .4-18$.

Cruz, A. (2011) La acumulación solidaria. Los retos de la economía asociativa bajo la mundialización del capital. Revista de Estudios Cooperativos, Unidad de Estudios Cooperativos - UdelaR, Vol. 16, No 1, pp. 12-37.

De Noble, A. F., Jung, D. y Ehrlich, S.B. (1999) Entrepreneurial Self-Efficacy: The Development of a Measure and its Relationship to Entrepreneurial Action. En Frontiers of Entrepreneurship Research 1999, editado por R.D. Reynolds, W.D. Bygrave, S. Manigart, C.M. Mason, G. D. Meyer, H. J. Sapienza, and K. G. Shaver, pp: $73-87$. Waltham, MA: P\&R Publications.

Duran, A.E y Arias, G. D. (2016) Actitud emprendedora y estilos emocionales. Contribuciones para el diseño de la formación de futuros emprendedores. Revista: Gestión de la Educación, Vol. 6, N 2, 83-102, ISSN: $2215-228$. Gestión de la Educación, Vol. 6, $\mathrm{N}^{\circ}$ 2, pp. 83-102. ISSN: $2215-228$. https://dialnet.unirioja.es/servlet/articulo?codigo $=5578742$.

Durán, A., E. (2013) Distinción entre actitud emprendedora y autoeficacia emprendedora: Validez y confiabilidad en estudiantes universitarios. Educación y futuro digital, $\mathrm{N}^{\circ}$ 7, pp. 59-69. Recuperado de: https://cesdonbosco.com/documentos/revistaeyfd/EYFD_7.pdf.

Encinas. M., L.C, (2018) Principales factores asociados al éxito o fracaso en el emprendedurismo. Revista de Investigación. Académica sin Frontera. ISSN: 2007-8870. Recuperado en: https://revistainvestigacionacademicasinfrontera.unison.mx/index.php/RDIASF/article/view/175.

Espíritu, R. (2011) Análisis de la intención emprendedora en estudiantes universitarios a través de los rasgos de personalidad. Multiciencias, 11 (1), pp. 65-75. Recuperado de: https://www.redalyc.org/pdf/904/90418851009.pdf.

Fundación AVINA. (2008) Liderazgo para el Desarrollo Sostenible en América Latina.

García, V., J.C., Villaseca, M., D. y González, P., S. (2019) Emprendimiento femenino y financiación social: un estudio comparado. REVESCO. Revista de Estudios Cooperativos, Tercer Cuatrimestre, $\mathrm{N}^{\mathrm{o}}$ 132, pp. 97-121. DOI: 10.5209/REVE.65974.

Gradl, C., y Knobloch, C. (2010) Inclusive Business Guide How to Develop Business and Fight Poverty Publication information. Retrieved from www.endeva.org.

Guerra, P. (2007) ¿Cómo denominar a las experiencias económicas solidarias basadas en el trabajo? Diálogo entre académicos latinoamericanos acerca de la polémica conceptual. Otra Economía, Unisinos, $2^{\circ}$ semestre, Vol. I, № 1 , pp. 21-27.

Guzmán. F., F.R., Llamas, E., M.L., Rodríguez, A., L y Alonso, C., M.M. (2014) Subjective norm, intention and consumption of marijuana in college students of México. Ciencia y Enfermeria. versión On-line ISSN 0717-9553. Vol. 18, No.1. http://dx.doi.org/10.4067/S0717-95532012000100006.

Hernández. R., C. y Arano. C, R.M. (2015) El desarrollo de la cultura emprendedora en estudiantes universitarios para el fortalecimiento de la visión empresarial, Ciencia Administrativa, No 1, pp. 28-38.

Hernández, S. R. y Mendoza, T. C.P. (2018) Metodología de la Investigación: Las rutas cuantitativa, cualitativa y mixta. Ciudad de México. Ed. Mexicana.

Hinkelammert, F. (2009) Economía para la vida. En Coraggio, J. L. et al. Diccionario de la otra economía. Buenos Aires: Universidad Nacional de General Sarmiento/Altamira/ Clacso.

Kramer, M.R. (2005) Measuring Innovation: Evaluation in the Field of Social Entrepreneurship. Skoll Foundation, Palo Alto, CA. 
Lara, G. (2011) Gestión de Empresas Sociales. Primera edición, México: Miguel Ángel Porrúa.

Lara, G., y Rico. A. (2011) La contribución de las cooperativas de ahorro y crédito al desarrollo local en Querétaro. $\begin{array}{lllllll}\text { REVESCO. Revista de Estudios } & \text { Cooperativos, } & \mathrm{N}^{\circ} & 106, & \mathrm{pp} . & 1-29 .\end{array}$ https://doi.org/10.5209/rev_REVE.2011.v106.37376.

Lupiáñez, L., P.T. y López., C. (2014) El emprendimiento como motor de crecimiento económico. Boletín Económico del ICE, del 1 al 28 de Febrero de 2014, pp. 59-61. Recuperado de: http://www.revistasice.info/index.php/BICE/issue/view/735.

Ley General de Sociedades Cooperativas (2018). Capítulo I Disposiciones Generales. Art. 6. Recuperado de: http://www.diputados.gob.mx/LeyesBiblio/pdf/143_190118.pdf.

Maestre, M., L.M., Páez., C, A.P., Mesías, F.J. y Lombana, C., J. (2019) Las cooperativas agrarias como modelo generador de negocios con inclusión social: el caso de las cooperativas bananeras del Magdalena (Colombia). REVESCO. Revista de Estudios Cooperativos, Tercer Cuatrimestre, No 132, pp. 195-217. DOI: 10.5209/REVE.65488.

Martinelli, A. (2004) The social and institutional context of entrepreneurship. International studies in entrepreneurship. Springer US, pp: 53-73.

Morales. J., J., Bustamante. G., A., Vargas. L., S., Pérez. R-, N. y Sereno. P, O. (2015) Factores de éxito emprendedor en dos municipios de la montaña de Guerrero, México. Revista Electrónica Nova Scientia, N ${ }^{\circ}$ 15, Vol. 7 (3), pp. 416-435.

Marulanda, J., Correa, G. y Mejía, L. (2009) Emprendimiento: Visiones desde las teorías del comportamiento humano. Revista Escuela de Administración de Negocios, $\mathrm{N}^{\circ}$ 66, pp. 153-168. Recuperado de: https://www.redalyc.org/pdf/206/20620269008.pdf.

OIT (2014) Cooperative movement engagement in sustainable development and the post-2015 process: Survey findings. Ginebra, OIT. Recuperado en: http://www.ilo.org/empent/Publications/WCMS_248497/lang-en/index.htm.

Pico, G.B., Vidal, A., Tapia, E. y Mendoza, A. (2019) Analysis of influence of Creativity and disruptive innovation one intention entrepreneur Case: México and Spain. Revista Contaduría y Administración. UNAM. Por publicar.

Prahalad, C. K., y Hart, S. L. (2002) Negocios en la base de la pirámide. Gestión, No 7, Vol. 3, pp. $108-117$.

Razeto, L. (2007) La Economía de Solidaridad: concepto, realidad y proyecto. En: Coraggio, J. L. (Org.) La Economía Social desde la periferia. Contribuciones latinoamericanas, Colección lecturas sobre Economía Social. Buenos Aires: Altamira, UNGS.

Rieiro, C., A. (2020) La economía y la recuperación del trabajo en Uruguay. REVESCO. Revista de Estudios Cooperativos, vol. 135, e69175.https://dx.doi.org/10.5209/reve.69175.

Rojas, J. J. (2003) Las cooperativas en México. México: Molino de Letras, Universidad Autónoma de Chapingo.

Salazar, E., Vargas, H., y Salazar, E. (Ed.) (2015) Investigaciones sociales en torno a la mercadotecnia y la sustentabilidad, México: Editorial: Competitive Press, S.A. de C.V.

Santos Araújo, F.; Nepomuceno, V.; Chedid Henriques, F., Pérez Pompeu, L., Mantovan Atolini, T. (Coords) (2019) Dialética da autogestao em empresas recuperadas por trabalhadores no Brasil. Marília: Lutas anticapial.

Shane, S., Locke, E.A. y Collins, C.J. (2003) Entrepreneurial Motivation. Human Resource Management Review, 13 (2), pp: 257-79.

Singer, P. (2007) Economía Solidaria. Un modo de producción y distribución. En: Coraggio, J. L. (Org.) La Economía Social desde la periferia. Contribuciones latinoamericanas, Colección lecturas sobre Economía Social. Buenos Aires: Altamira/UNGS.

SNV (2008) - Servicio Holandés de Cooperación al Desarrollo, y CECODES - Consejo Empresarial Colombiano para el Desarrollo. (2008) Los Negocios Inclusivos en Colombia. Bogotá D.C., Colombia.

Sommers, G. (2008) El cooperativismo y las cooperativas pesqueras del Soconusco. Antecedentes, desarrollo y praxis. Recuperado de: http://www.eumed.net/libros/2012a/1154/cooperativismo_y_cooperaticas_pesqueras.html.

Torres, J. M.E., y Contreras, L., M.R. (2013) Las organizaciones cooperativas: el proceso administrativo como parte de la gestión directiva. Journal of Intercultural Management. Vol. 5, No. 1, pp. 51-61. DOI 10.2478/JOIM-2013-0004.

Urban, B. (2013) Social Entrepreneurship in an Emerging Economy: A Focus on the Institutional Environment and Social Entrepreneurial Self-Efficacy. Managing Global Transitions, 11 (1), pp: 3- 25. 